\title{
Sensor, Signal and Image Informatics
}

\section{State of the Art and Current Topics}

\author{
T.M. Lehmann ${ }^{1}$, T. Aach ${ }^{2}$, H. Witte ${ }^{3}$ \\ 'Department of Medical Informatics, Aachen University of Technology (RWTH), Aachen, Germany \\ Institute of Imaging and Computer Vision, RWTH Aachen University, Aachen, Germany \\ ${ }^{3}$ Institute of Medical Statistics, Computer Sciences and Documentation, Friedrich Schiller University \\ Jena, Jena, Germany
}

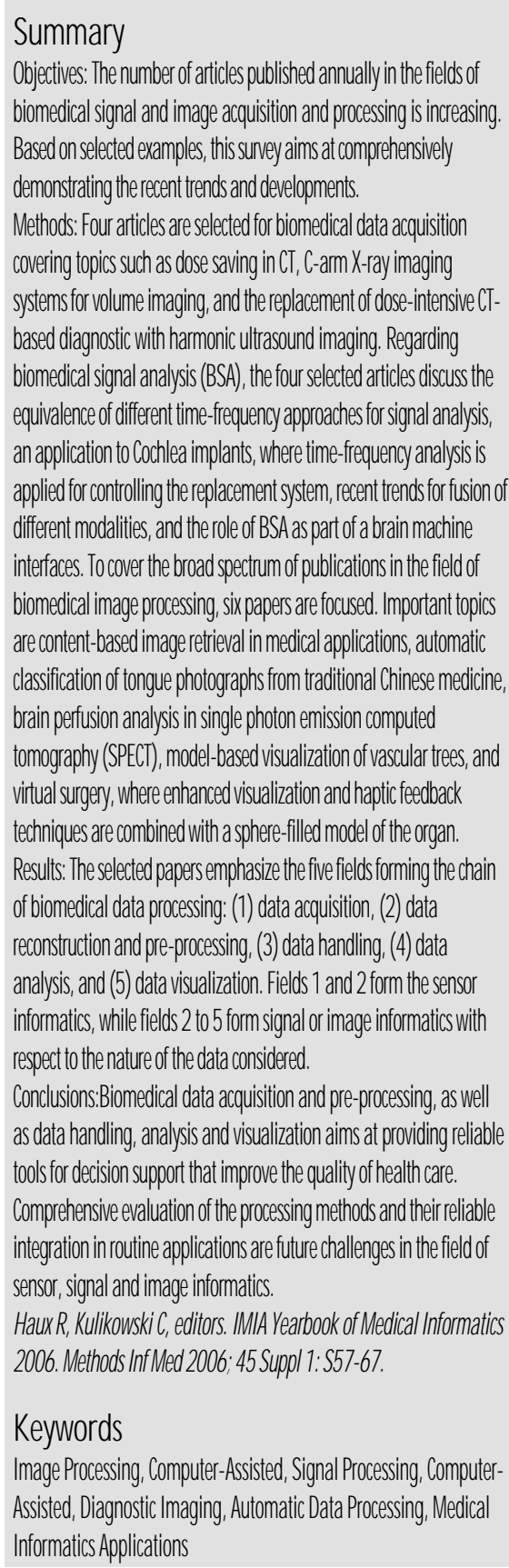

\section{Introduction}

The past, present and future of biomedical imaging informatics were analyzed in a recent review [1]. Reflecting on the paradigms of quality management where structure, process and result quality are analyzed separately, it was found that most infrastructure- and processing-pipeline-related problems have already been resolved. Therefore, the future of biomedical data processing was seen to be linked to the results obtained from the methods applied in medical informatics. Since these results are measured as the direct outcome for the patient, biomedical sensor, signal and image informatics is worthless unless it is capable of being applied routinely and to significantly improve the patient's healthcare.

Figure 1 sketches the chain of biomedical data processing. Induced by the physicians or the patients themselves, the data is acquired and preprocessed, which results in the biomedical signal or image. This part of the chain is referred to as sensor informatics. Data management (e.g., storage, retrieval and communication), data visualization (e.g. transforms and appropriate displaying for qualitative inspection by human observers), and data analysis (e.g. quantitative measurements that are automatically determined by computers) are termed signal or image informatics with respect to the technical nature and dimensions of the acquired data. Processing a set or map of onedimensional (1D) electrical signals captured continuously over time is called biomedical signal informatics, while the processing of two-dimensional (2D) spatial data, volumes (threedimensional, 3D), or image and volume sequences (four-dimensional, 4D) is referred to as image informatics. Both aim to support decision making and improve the patient's healthcare. Furthermore, the development and integration of robust applications was identified as the next major challenge to face [1]. Without claiming completeness, this paper exemplarily surveys recent developments towards this goal in the fields of sensor, signal and image informatics. (References to these papers are typeset in bold face.)

\subsection{Sensor Informatics}

The acquisition process is the first part and thus a key issue in the chain of medical imaging. The performance of the image acquisition process in today's systems often depends on a sophisticated interaction between the sensors themselves and the subsequent signaland image processing components (Fig. 1). These processing components comprise algorithms to compensate for various known, and often calibrated, sensor imperfections, such as varying offset and sensitivity of pixels of a flat 


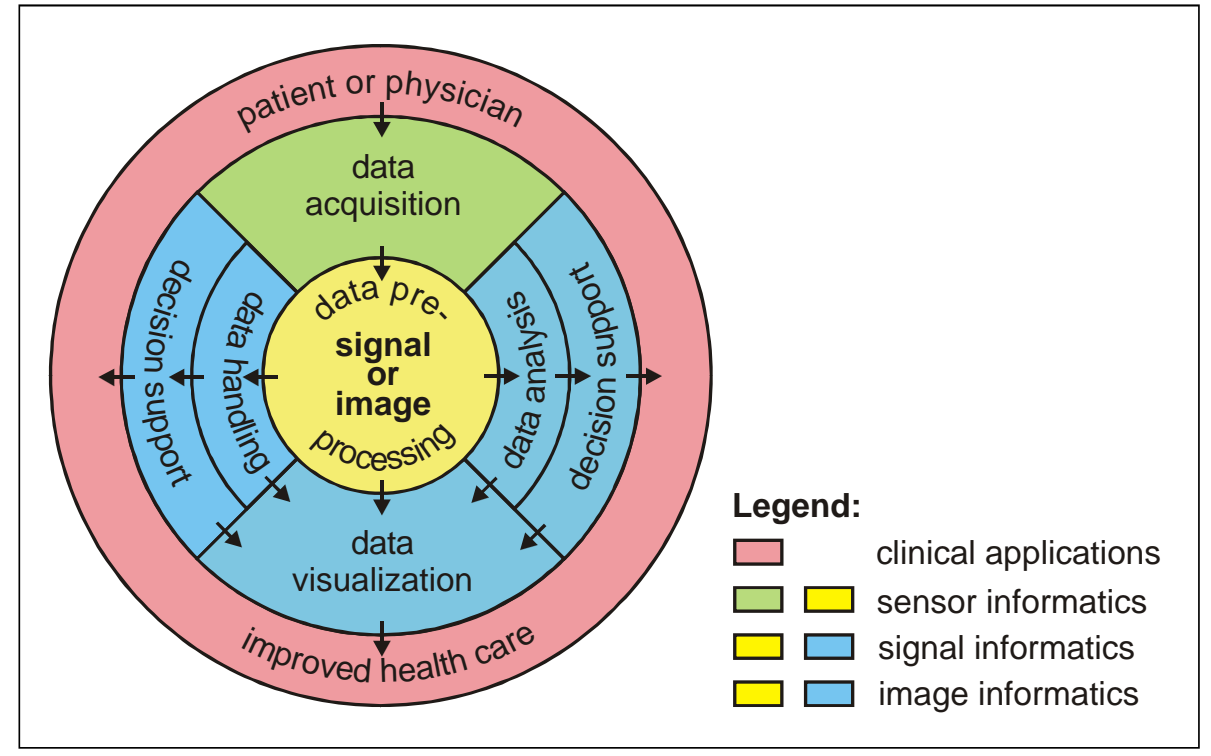

Fig. 1 Sensor, signal, and image informatics embedded in dinical applications

solid-state $\mathrm{x}$-ray detector, or interpolating across inactive pixel sites. On the next higher level, these components include algorithms to convert measured data into images, e.g. homographic reconstruction, hybrid tuned-aperture computed tomography (TACT $\left.{ }^{\circledR}\right)$ [2], limited angle 3D digital subtraction angiography (DSA) [3], formation of ultrasound brightness (B)-scans from the envelope backscatter signals of a transducer, or novel methods such as cardiac activation time imaging [4]. Finally, the image acquisition process may be controlled adaptively depending on the data recorded, for instance to reduce $\mathrm{x}$-ray dose in CT imaging as described below. We focus on the probably most widely used basis for medical imaging, viz. $x$-radiation, with its well-known trade-off between image quality and potentially harmful effects to patients and medical staff. In the following, we therefore discuss approaches to reduce the $\mathrm{x}$-ray dose in $x$-ray volume imaging, or even to replace this with other imaging methods such as ultrasound.

\subsection{Signal Informatics}

The techniques for biomedical signal analysis (BSA) have evolved from time-invariant to time-variant processing [5], from univariate to multivariate [6, 7], from frequency to time-frequency [8], and from linear to non-linear analysis $[9,10]$. Time-variant, multivariate linear and nonlinear analysis methods are currently in use and their application can be shown. Additionally, the trends from off-line to on-line analysis and from processing one modality (signals) to different modalities (signals and images) can be observed [11]. Furthermore, signals have been analyzed to characterize physiological systems that generate them. Therefore, system identification can be seen as one major objective of BSA [12].

The fundamental driving forces behind these changes have been developments in sensor technology and the necessity for improved signal interpretation [13]. All recent progress in BSA are due to technological developments which themselves have been powered by the user's or customer's options. As mentioned before, the goal of biomedical signal analysis and its interpretation is to assist clinicians in their decision making. Medical practitioners, clinicians and biomedical researchers use BSA such that the patients ultimately benefit from improved prevention, diagnosis, therapy and rehabilitation (Fig. 1). For these purposes, improvements in all steps of BSA are necessary:

1. preprocessing and providing data validity (e.g. calibration, filtering, trend removal and artefact detection [14]) as well as combining different data modalities (e.g. fusion of electroencephalography (EEG) and functional magnetic resonance imaging (fMRI) data [11]);

2. data management (e.g. compression [15] or fusion [16]);

3. parameter extraction, data reduction and visualization (e.g. [17]);

4. data analysis such as system identification and modeling (e.g. source modeling on the basis of EEG, magnetoencephalography (MEG) or electrocardiography (ECG) data, model-based processing of cardiovascular data), classification and pattern recognition aiming at prediction or knowledge-based decision making (e.g. fused data analysis [18], fusion of knowledge and combination of processing results $[19,20])$.

These steps have been developed differently in each BSA strategy and the contribution of each step to the complete processing chain is weighted differently. The challenge of BSA is the automation of biosignal interpretation by the enhanced combination of signal processing (up to the step of parameter extraction), pattern recognition and knowledge-based decision making (artificial intelligence [20]) as well as the statistically founded evaluation of such systems [21].

The most pressing motivation for automating biosignal interpretation arises 
Sensor, Signal and Image Informatics

due to human factors such as problems of data overload, necessity of an immediate reaction time by imminent danger, varying expertise and human error. Automated biosignal interpretation can assist clinicians in the task of interpretation itself to aid in avoiding errors in diagnosis and the selection of treatment. In biomedical research, an appropriate signal interpretation enhances the understanding of fundamental observations and measurements.

\subsection{Imaging Informatics}

In the early years, information logistics was the primary aim of any medical information system [22]. In particular, the paradigm was postulated that the right information has to be delivered at the right time and in the right place [23]. In picture archiving and communication systems (PACS), this medical information is the patient's image data or its derivatives. Today's PACS are based on the digital imaging and communications in medicine (DICOM) standard that allows the connection of imaging devices, computers for data analysis, viewing stations, and huge image archives with the radiological information system (RIS) hosting the corresponding patient records. Fast networks support the rapid transfer of image data within the hospital to (even portable) high-quality display devices, and the Internet is used to interconnect between healthcare providers. The emerging number of digital imaging modalities with steadily increased resolutions has transformed PACS archives from a simple library of images into a medical knowledge repository that can considerably benefit research, teaching, and diagnostics. Data handling, visualization and analysis are therefore the major fields for computer applications in image informatics (Fig. 1).

\section{Sensor Informatics}

$\mathrm{X}$-ray volume imaging and transcranial ultrasound are two modalities where visible technical developments and improvements were recently achieved.

\subsection{X-Ray Volume Imaging}

\section{Dose Reduction by Tube Current Modulation}

The traditional method of $x$-ray volume imaging is fan beam computed tomography (CT) $[24,25]$. With the advent of the multi-detector CT [26], new time-resolved CT imaging has become possible, for instance CT angiography and cardiac CT imaging.

A critical issue, however, is the still relatively high patient radiation dose associated with $\mathrm{CT}$ imaging [27]. In quantum-limited $\mathrm{x}$-ray imaging, both the variance of the detected noise and the signal-to-noise ratio (SNR) decrease linearly with decreasing impinging quantum flux [28]. Areas in the projection images corresponding to high attenuation thus exhibit low SNR, an observation which also holds after nonlinear conversion by taking the logarithm to obtain the line integrals. With respect to CT imaging, this implies that projections taken under angles for which most $\mathrm{x}$-rays travel a relatively long way through the patient (e.g., lateral projections) exhibit lower SNR than those projections where the travel paths are short (e.g., anteroposterior (AP) projections). Therefore, one approach towards reduction of patient dose without compromising the image quality is to vary the applied dose depending on the total patient attenuation for each angle [29], with a considerable dose reduction for AP-projections. Based on modeling the patient's body by an ellipse, this dose variation may be carried out by a sinusoidal modulation of the tube current over projection angle, with the modulation amplitude being determined off-line by a priori lateral and AP localizer projections [30].

Today, rather than using a parametric function of dose vs. angle, with the parameters being determined off-line, the dose may be adapted on-line to the patient's attenuation for each projection angle. For multi-slice $\mathrm{CT}$ imaging, the clinical study described in [31] reports dose savings of about $30 \%$, without compromising diagnostic image quality: While noise increased only statistically insignificantly, artefacts such as streaking can even be reduced by a slight increase of tube current for lateral projections, and a corresponding considerable decrease for AP projections.

\section{Projection Interpolation for Cone-beam CT}

The availability of fast reconstruction algorithms for cone beam CT [32, 33] has made it possible that $\mathrm{x}$-ray volume imaging can today also be performed by interventional $\mathrm{C}$-arm-based systems, which are predominantly used for interventional $\mathrm{x}$-ray imaging such as fluoroscopy and cine-angiography. Equipped with image-intensifier and camera detection front ends [28, 34, 35], interventional $\mathrm{x}$-ray systems were first applied in the reconstruction of vessels filled with a radio opaque contrast agent ("rotational angio") [36]. The projections are acquired by rotating the $\mathrm{C}$ arm gantry around the patient, and recording the 2D attenuation images. The acquisition of 2D projection images instead of single-slice or multi-slice 1D projections as in conventional CT extends the fan beam towards a cone beam, and necessitates a corresponding extension of reconstruction algorithms. With the newer generation of solid state flatpanel radiography detectors [37] possessing a larger dynamic range than 
image intensifier-based front ends, volume reconstruction was considerably improved, allowing the imaging of objects with less contrast [38, 39, 40]. Mechanical and frame rate limitations may, however, lead to an only relatively low number of angular cone beam projections being recorded. Volume data reconstructed from such sparsely sampled angular projections exhibit artefacts such as streaking and noise.

To reduce or even eliminate these artefacts, in [41] an approach is described to increase the number of projections by interpolating between the available ones. To avoid blurring, the developed interpolation scheme is specifically geared towards 3D sinograms. The basic idea is to adapt interpolation to the local information content based on detecting and estimating oriented structures, and to weight the interpolation such that information from the detected orientation, if any, has a stronger influence. Orientation is detected and estimated by an eigensystem analysis of an extended version of the tensor developed in [42, 43], with the eigenvalues indicating whether or not a uniquely identifiable orientation is present, and the eigenvectors providing information about the orientation itself. Results obtained for simulated sinogram data of an anthropomorphic head phantom show that the nonlinear directional interpolation method indeed reduces streaking artefacts and noise, and outperforms other interpolation methods such as linear, cubic spline-based and regularized interpolation [44, 45].

\section{Scatter Compensation for Cone-beam CT}

The quality of volume data from $\mathrm{C}$ arm-based cone-beam CT is not only influenced by the dynamic range of the detectors used and angular sampling density, but also by scattering [46]. Artefacts from scatter in cone-beam CT include streaking, noise, an inhomogeneity-like effect of low spatial frequency content termed "cupping", and limitations on soft tissue contrast resolution. Compared to fan-beam CT, the effects of scattering are much more severe due to the larger volume irradiated by the cone beam, which causes a higher amount of photons to be scattered.

The use of anti-scatter grids does not achieve the desired effect of decreasing the scatter-to-primary radiation ratio, since for the geometry of C-armbased cone-beam CT, the scatter attenuation is outweighed by unavoidable absorption of primary radiation [47, 48]. Computer-based a posteriori scatter correction schemes, which estimate and compensate scattered radiation, are therefore needed.

One such technique, which is based on a 3D model approximating the imaged part of the human body, is developed and evaluated in [49]. The approach takes into account single and multiple scattering events as well as the polyenergetic nature of $\mathrm{x}$-radiation. To circumvent the exponentially increasing computational expense for the estimation of multiple scattering, only single scattering is calculated accurately, whereas multiple scatter background is obtained by a parametric model from the estimated single scatter. For single scatter estimation, the 3D anatomical model is sampled to a voxelized representation, and the total scatter contributed to each detector pixel is computed by adding the individual contributions from each primary ray. These individual contributions, in turn, are calculated from the scattering probability at each voxel and the probability that the scattered photon leaves the scattering site under a certain angle. The performance of the model-based scatter estimation approach is shown for a 3D water-filled ellipsoid serving as an approximation of a human head. Ground truth data is generated by computationally very expensive Monte Carlo simulations. Highly accurate estimates of the scattered radiation were achieved. Compared to reconstruction from uncompensated projections, artefacts could be considerably attenuated. For instance, the amplitude of the above-mentioned cupping artefacts was reduced more than ten-fold from 250 Hounsfield units (HU) to about $20 \mathrm{HU}$ [49]. The computational costs of this approach are sufficiently low such that the approach is suitable for scatter compensation in practice.

\subsection{Stroke Diagnostics by Trans- canial Ultrasound Harmonic Imaging}

The therapeutic success of treatment of patients with acute ischemic stroke requires an early and reliable diagnosis of brain areas with critically reduced perfusion. Towards this end, visualizing cerebral micro-perfusion is crucial. Because of its high diagnostic image quality, cranial CT (CCT) is, besides magnetic resonance tomography and nuclear imaging techniques, one of the diagnostic methods used in routine protocols for stroke patients. However, apart from the above mentioned exposure of the patient to relatively high radiation doses when using CCT, it also has the drawback of being time-consuming and expensive, and not being well suited for restless or critically ill patients who have to be transported to the imaging system.

In contrast to this, ultrasound imaging is a comparably inexpensive, fast and well tolerated bedside imaging method. However, since the assessment of brain perfusion implies insonation through the skull with its high acoustic imped- 
Sensor, Signal and Image Informatics

ance, conventional transcranial colorcoded sonography requires a sufficient acoustic bone window [50]. An alternative is so-called harmonic imaging using an ultrasound contrast agent, such as SonoVue, which is a sulfurhexafluoride-containing aqueous suspension of phospholipid microbubbles. Such contrast agents are highly resonant at diagnostic ultrasound frequencies and, more importantly, generate harmonics of the insonation frequency, which make the response distinguishable from tissue response [51, 52]. The contrast between perfused and non-perfused areas is thus enhanced.

The studies in $[\mathbf{5 0}, 53]$ describe an approach towards tracking a bolus injection of ultrasound contrast agent (bolus harmonic imaging, BHI) and visualizing its kinetics qualitatively. Its clinical significance is also reported. The analysis of the flow kinetics is based on four parameter images, depicting (i) pixel-wise peak intensity, (ii) time to peak, (iii) area under curve and (iv) average slope. For each of 23 patients, the investigation was carried out within an axial midthalamic plane of the symptomatic brain hemisphere defined by the third ventricle, the thalamus, and the anterior horn of the ipsilateral ventricle. Insonation and readout were performed using a $1.8 / 3.6 \mathrm{MHz}$ sector transducer. It could be shown that the area of significant amplitude decrease detected in the early phase of ischemic stroke exhibits significant correlation with the definite area of infarction shown in follow-up CCT and with patient outcome after four months. The study thus demonstrates the clinical relevance of $\mathrm{BHI}$, showing that it increases the diagnostic relevance of neurosonology by providing additional information obtained at the patient's bedside in the early phase of ischemic stroke.

\section{Signal Informatics}

The device-related applications of BSA for patients and handicapped persons need, firstly, a profound understanding of fundamental biomedical functions and, secondly, a comprehensive and appropriate integration strategy for all technical components, i.e. from the sensors to decision making or actuators (inclusive stimulation, robot control etc.). Major applications of BSA within this framework are:

- Automatic triggering and control of medical devices (e.g. event-related triggering of MRI [54], automated infusion control [55], adaptive control of artificial heart pace-makers);

- Monitoring and support of patient's treatment as well as rehabilitation (e.g. intensive care monitoring, signal-assisted biofeedback strategies, and functional electrical stimulation [56]);

- Substitution as well as replacement systems (e.g. sensory substitution systems $[57,58]$, brain computer, or brain machine interfaces $[\mathbf{5 9}, 60])$.

The following studies were chosen to demonstrate these kinds of developments in BSA technology and potential applications.

\subsection{From Frequency to Time- frequency BSA - The Equivalence of Methods}

The developments of BSA itself towards time-variant (time-frequency), multivariate analysis methods have resulted in a number of different approaches. Time-frequency approaches commonly used are:

1. Time-frequency distributions (e.g. short-time Fourier transform, Wigner-Ville spectrum);

2. Wavelet transform;

3. Hilbert transform;
4. Time-frequency representations based on time-variant parametric models.

With these methods (and other methods, too) the possibility of expanding to a bivariate time-frequency analysis exists, i.e. cross-spectral, cross-phase and coherence analysis. Multivariate, time-variant parametric models enable multivariate approaches. Bruns [61] demonstrated that Fourier-, Hilbertand wavelet-based signal approaches are equivalent if a certain window function (Fourier-), a certain filter (Hilbert-), and a certain kernel envelope (wavelet-transform) are used. Unfortunately, a common flaw of previous comparative studies has been that the approaches have not been matched regarding timefrequency resolution. The paper begins with the definition of spectral parameters and derived measures which are frequently used in time-frequency analysis of neurophysiologic signals, i.e. time evolution of amplitude (envelope), phase and coupling measures (bivariate). A formal (mathematical) and an empirical (using spectral parameters) comparison of the approaches show that it is of minor importance which approach is used. Important is to use those approaches which have the optimization capability to permit an optimal detection of defined signal properties which are expected in biosignals. Simulations of expected properties are helpful to optimize the parameters of the analysis method.

\subsection{Time-frequency Analysis for Controlling Replacement Systems- Cochlear Implants}

Nie et al. [62] demonstrate a particular approach of time-frequency analysis, which is comparable to a filter-based 
Lehmann et al.

Hilbert transform using this algorithm to control a cochlear implant. Sensory substitution devices allow a remaining sense to take over the functions lost as the result of the sensory impairment. Sensory correction and replacement are alternatives to sensory substitution. Cochlear implants replace much of the function of the cochlea with direct electrical stimulation of the hearing nerve. Persons who become deaf usually lose only the peripheral structures relating to sound transduction (the cochlea). The implants differ in the way that they process sound and how they present electricity to the hearing nerve. Cochlear implants can be distinguished by their way of encoding sound information (analog and digital coding) and by their use of single vs. multiple channels, the number of electrodes, and their use of either mono-polar or bipolar stimulation. Additionally, there are many different ways of processing the auditory signal for presentation at the level of the cochlear ganglia. But a common property of all methods is the use of different numbers of band-pass filters and information content. For example, the continuous interleaving sampled (CIS) system uses 4-22 frequency bands. The processing and conditioning of the speech signal is carried out by a speech processor, i.e. a specialized digital signal processor.

Narrow band-pass-filtered signals can be described as a simultaneously amplitude and frequency (phase) modulated signal. Therefore, demodulation techniques, e.g. using the Hilbert transform, are frequently applied to signals with narrow band characteristics. The instantaneous amplitude (envelope), frequency and phase can be computed and lead to special time-frequency representations in contrast to spectrogram-like time-frequency plots. Nie et al. [62] use a new frequency-amplitude- modulation-encoding algorithm to improve cochlear implant performance in realistic listening situations, i.e. in a noisy environment. Cochlear implants use speech processing strategies that focus on extracting (analysis) and representing the amplitude modulation cue. They evaluated the values of amplitude and frequency modulation of band pass filtered speech sound signals, where amplitude modulation properties are frequently used for the conditioning of stimulation (see above CIS). The authors use a phase vocoder (name derived from voice decoder) technology designed by Flanagan [63]. The results demonstrate the complementary contribution of amplitude and frequency modulation to speech perception. Amplitude demodulation from several frequency bands is sufficient to support speech recognition in quietness, and the frequency demodulation properties are needed for speech recognition in noise, in particular, when the noise is a competing voice reflecting more realistic listening situations [62].

\subsection{The Compensation of Lost Voice by BSA - Fusion and Analysis of DifferentData Modalities}

The replacement of the sound-generating function of an excised larynx by a natural substitute (mucosal tissue at the upper part of the esophagus) allows a compensation of the lost voice with a high quality. Substitute voice quality needs further research. Lohscheller et al. [18] introduced an analysis strategy to quantitatively analyze the vibration pattern of the substitute and to investigate its relation to the emitted acoustic signal. They simultaneously recorded the acoustic signal with a microphone and the vibration patterns of the substitute with a digital high-speed camera (placed into the oropharynx). The combination of image processing of highspeed image sequence analysis and the analysis of the acoustic signal is one key feature of the evaluation algorithm. The detection of region of interest (ROI) uses three image features to determine the contribution to the sound generating process and these three criteria are merged to a normalized ROI. The first criterion is based on the computation of the maximum of the normalized cross-correlation between the image intensity at each pixel position and the acoustic signal. A region-growing algorithm was used for the segmentation of the ROI. After initial determination of the ROI's contour line, a contour tracking follows to derive binary edge maps which contain the ROI evolution in time, i.e. the algorithm consists of an initialization and an object tracking part. In this way, the ROI's area integral can be computed and quantitatively analyzed as a function of time $a(t)$ for each high-speed sequence, i.e. signal analysis algorithm can be applied. The $a(t)$ signal for each of the three image sequences and the acoustic signal were analyzed by spectral analysis (amplitude spectra via Fourier transform). This study is an outstanding example of the fusion and analysis of different data modalities by means of BSA.

\subsection{BSA as a Part of a Brain Machine Interface - Brain Adivity Controls a Robot}

Patients may employ EEG feedback training to influence their own brain activity (self-regulation). Such "learned" EEG patterns can be used to control an endogenous brain computer interface (BCI) or a brain machine interface (BMI). It was shown that a paralyzed patient learned to "produce" distinct 
Sensor, Signal and Image Informatics

EEG patterns by mental imagery and to use this skill for BCI-controlled spelling. But the training period required several months. Such self-regulation of EEG rhythms (or potentials, patterns, properties) can be learned through operant conditioning (according to Skinner's concept that "the behavior is followed by a consequence, and the nature of the consequence modifies the organism's tendency to repeat the behavior in the future."). The known EEG activities which can be self-regulated are: event-related potentials (ERPs), slow cortical potentials (SCPs), and special EEG frequency components.

Within this framework, del R. Millan et al. [59] demonstrated that a continuous control of a miniature robot in an indoor environment is possible by the recognition of special mental imageries of a subject via 8-channel EEG analysis (time-invariant spectrum analysis), parameter extraction (12 frequency components), and a subsequent classification (statistical classifier). The subjects trained for the following mental tasks: "relax", imagination of "left" and "right" hand movements, "cube rotation", "subtraction" and "word association". After an EEG feedback training period, subjects learned to mentally control the robot. The robot itself had 6 perceptual states (open space, obstacle to left, obstacle to right, wall to left, wall to right, wall in front). The precondition for a control scheme is the mutual connection of mental states of the subject with the perceptual states of the robot, i.e. the robot's interpretation of the mental imaginary must be dependent on the perceptual state of the robot. To fulfill this aim, a finite state automaton was used. Another problem arises. Other sources with influence on the control process must be excluded. Therefore, the influence of eye movements (electrooculogram, EOG) and facial electromyographic activity (electromyogram, EMG) must be rejected. Additionally, it can be expected that subjects may use EOG and EMG activity as the control signal (one man's artefact is another's signal). The authors ruled out the influence by the use of frequency ranges $8-30 \mathrm{~Hz}$ (EOG occurs in the range $<4 \mathrm{~Hz}$ ) and electrodes (EMG artefacts are more prominent in anterior electrodes). These results demonstrate the strong feasibility and significant potential benefit offered by the concept of controlling robots or prosthetic devises by mental imaginary with the help of BSA.

\section{Image Informatics}

For each of the major fields within the chain of data processing (Fig. 1), we have selected an exemplary remarkable approach that has been developed and recently published.

\subsection{Data Handling}

Clinical decision support techniques such as case-based reasoning or evidence-based medicine require access to images and corresponding medical records from more than one patient or from different studies. In today's PACS, these needs are insufficiently supported since access to all image data is based on textual (i.e. alphanumerical) descriptions only. Content-based image retrieval (CBIR) is a field of research and applications that attempts to augment text-based search by the incorporation of visual information analysis. For instance, the integration of content-based methods into a PACS allows automatic comparison of the image to be diagnosed with all earlier cases stored in the archive. For that, a sample image or image region is presented to the system, which answers this query by returning all similar matches. This concept is referred to as query by example (QBE). It was introduced in the early 1990s by Niblack et al. presenting IBM's query by image content (QBIC) system $[64,65]$. Since color was found to be the most discriminating visual feature, CBIR was not used in the medical domain. However, almost ten years ago, Tagare et al. have foreseen that integrating CBIR into clinical routine will significantly improve the quality of patient care if local regions and their spatial or temporal relationships are analyzed [66].

Müller et al. have recently reviewed CBIR systems in medical applications regarding their clinical benefits and future directions [67]. Still, the major problem is to close the semantic gap between image similarity defined by humans on a high-level concept of semantics such as local object identification and scene analysis, and image similarity computed automatically using the low-level pixel information [68]. Therefore, existing systems are usually restricted to a certain imaging modality and body region, and they are usable only in a well defined context of medicine. Radiology, pathology, and dermatology are the most frequent fields of applications.

Müller et al. particularly referred to the image retrieval in medical applications (IRMA) approach [69] as one of the rare medical CBIR approaches for general use. In order to close the semantic gap, levels of information abstraction are defined in IRMA and computed sequentially. In the first stage of feature extraction, global texture descriptions are computed from the QBE pattern and used to determine the body region (Anatomy) and biomedical system (Biology) imaged, the imaging modality 
and technique (C-reation), and the relative orientation between the patient and the imaging device (D-irection) [70]. These preprocessing steps allow to automatically adapt subsequent local image analysis to the medical context, which is unknown before the user has formulated the query.

In [71], an approach for automatic categorization of medical images is introduced and thoroughly evaluated. Here, categorization means selecting the appropriate class for a given image out of a set of pre-defined categories. In total, 81 categories were pre-defined using the mono-hierarchical multi-axial nomenclature of the IRMA ABCD-code [70]. A set of 6,231 images was selected arbitrarily from clinical routine, and each image was annotated by experienced radiologists with its corresponding unambiguous IRMA code. This testbed was used to determine the correctness of automatic categorization by numerous leaving-one-out experiments. The visual properties were assessed combining textural and correlation-based features. The textural features are based on the fundamental work of Haralick et al. [72]. Tamura et al. suggested coarseness, contrast and directionality to describe an image's texture properties [73], which are formed into a histogram of 384 bins [74]. These features were combined with the image distortion model (IDM). IDM is a correlationbased measure that additionally allows local displacements for each pair of corresponding pixels [75]. This is especially useful for medical images due to their individual anatomical or pathological properties. The IDM measure is computed based on vectors of identical size $h \times h, h \in\{8,16,24,32\}$, to which the images were scaled ignoring their original aspect ratio. In other words, only 64 to 1,024 numbers were used to represent the entire medical image.
The best correctness of $85.5 \%$ is obtained for $h=32$. However, for the CBIR-related task of retrieving the correct class within the ten best matches, the best rate of $97.72 \%$ is obtained for $h=24$. Here, an image is reduced to a combined feature vector formed from less than 1,000 bins. Regarding the difficulty of the categorization tasks, which is caused by high intra-class variability and high inter-class similarity as well as the large differences of the sample sizes in the reference categories, these results are absolutely remarkable. Furthermore, similarities of anatomical regions (e.g. elbow vs. knee), biomedical systems (e.g. in radiographs of the abdomen), imaging modalities (e.g. the use of collimation fields and shutters), as well as imaging directions (e.g. craniocaudal vs. oblique views in mammography) may be assessed by subsequent classifiers that are especially designed for each of the tasks (e.g. [76]).

\subsection{Data Analysis}

If trained for a certain imaging modality, image similarity measures can also be used for computer-assisted diagnosis. For instance, Pang et al. have presented a system for computerized tongue diagnosis [77]. In traditional Chinese medicine, tongue diagnosis is concerned with the identification of syndromes rather than with the connection between tongue abnormal appearances and diseases [78, 79, 80]. In contrast, the novel method that is based on texture and chrominance measures obtained from different parts of the tongue is dedicated to the classification of 14 diagnostic categories (13 common diseases and healthy). In total, 455 patients were analyzed. Using a Bayesian network classifier, a prediction accuracy of $75.8 \%$ is reported.
Likewise in this example, automatic image analysis in general aims at providing quantitative measures to support decision making. According to [1], registration (e.g., [81]) and segmentation (e.g., [82, 83, 84]) currently are active fields of research. However, such algorithms must be thoroughly evaluated using a sufficiently large number of images. Frequently, images with apriori known ground truth are unavailable and consequently, such a computerized method lacking statistically founded evaluation is not used in clinical routine.

Imabayashi et al. [85] have presented an evaluation of computerized brain perfusion in single photon emission computed tomography (SPECT) analysis that is capable of establishing the method in clinical routine. In Alzheimer's disease (AD), regional cerebral blood flow (rCBF) in the posterior cingulated gyri and precunei has been reported to decrease even at a very early stage. Visual inspection of SPECT images is compared to automatic analysis of atlas-registered images, where the volume data is projected on 3D stereotactic surfaces [86, 87]. In total, 38 patients with probable $\mathrm{AD}$ were imaged at a very early stage and after a mean interval of 15 months. The data is compared to that of 76 age-mapped healthy volunteers. The subjects were randomly divided into two groups. The first group was used to identify areas of decreased $\mathrm{rCBF}$ in the 3D surface projections. The second group was used to evaluate the automatic method with visual inspection. Six trained physicians graded the rCBF decrease on SPECT images for receiver operating characteristic (ROC) curves. Each observer inspected the images twice at an interval of at least two weeks.

Visual inspection showed fair-to-excellent intra- and inter-observer reliabili- 
Sensor, Signal and Image Informatics

ties. Automatic analysis of surface projections demonstrated an accuracy of $86.2 \%$. In contrast, the accu.racy of visual inspection was below $74 \%$.

\subsection{Data Visualization}

According to Figure 1, data visualization is an important step in medical signal and image analysis. Consequent$1 y$, numerous papers on the visualization of medical data and related fields are still appearing. In particular, the performance of $3 \mathrm{D}$ visualization is continuously enhanced with respect to both speed and quality.

For instance, it had been shown that vessel trees, which have been segmented from individual CT or MRI data, can be visualized more realistically if the segmented volume data is not rendered directly, but the visualization is based on model data extracted from the segmented voxels [88]. Advantageously, segmentation errors immediately become obvious. Assuming circular vasculature, vessel trees can be described by their skeleton and represented as a directed graph attributed with the local diameter, which is determined by image analysis [89]. Photo-realistic rendering is obtained if this implicit modeling is smoothed and combined with so called convolution surfaces [90]. Based on convolution surfaces, Oeltze and Preim take special care at branchings to weight the incident branches [91]. The improved visualization quality is demonstrated qualitatively, and evaluated both qualitatively by 11 observers as well as quantitatively by means of simulated data.

Beyond the photo-realistic visualization of existing structures, simulation tackles the visualization of altered structures. Frequently, methods of virtual reality are applied and haptic feedback is used to complete an immersive impression. For instance in the paper by
Suzuki et al., a virtual surgery system is presented that is capable of simulating surgical maneuvers on elastic organs [92]. While finite element methods (FEM) have been applied so far, which are capable of performing (i) realistic, (ii) real time, and (iii) quantitative deformations, the novel approach of Suzuki et al. is additionally capable of (iv) representing internal structures of organs, (v) simulating various forms of manipulations such as movement, deformation under pressure, incision and partial re-movement, and (vi) enabling the easy computation of force feedback.

The basic idea is to fill the triangulated surface by elementary spheres, and calculate the movement of the spheres with respect to a model of gravity. Inherently, the volume of the deformed or altered soft tissue organ is kept constant because rigid spheres are modeled. Furthermore, the authors have equipped their model with a sense of touch and a sense of force by connecting it to a force feedback device. Suzuki et al. have applied their model to simulate a life liver donation procedure, where the resection of the donator's liver tissue is about to be implanted into the receiver. In particular, the force feedback that can model five fingers of each hand was evaluated in vitro using a slice of organ and a compression device. According to the authors, the simulation was confirmed by an experienced surgeon. Using a radius of $8 \mathrm{~mm}$ for the spheres, a frame rate of $25-30 \mathrm{fps}$ is obtained on an Octane 2 graphic workstation (Silicon Graphics Inc).

Based on the sphere-filled model of organs, other surgical techniques such as resection and grasping should be able to be performed. Although the system is designed to simulate the homogeneous properties of organs, it is also capable of coping with inhomogeneous prop- erties if the internal motion of the elementary spheres is modified. This should allow, for instance, to realistically simulate tumor-indicated procedures.

\section{Condusion}

Sensor, signal and image informatics are emerging fields of computer science in medicine. Based on recently published selected examples, we have shown how a carefully designed interplay between the imaging sensors, their control and subsequent processing leads to improved or even new diagnostic methods for the benefit of the patient. The survey also includes recent trends in biomedical signal and image analysis, and the discussed applications demonstrate that new methods are integral parts of the biomedical data processing and analysis chain in medicine (Fig. 1). Additionally, it can be shown that the adaptation of methods to special signal properties and biomedical application requirements is an indispensable step of the design of processing procedures. However, the underlying aim is still that the patients ultimately benefit through improved methods in diagnosis, therapy and rehabilitation.

\section{References}

Exemplary papers that are certainly discussed in this survey are type-set bold face.

1. Lehmann TM, Meinzer HP, Tolxdorff T. Advances in biomedical image analysis - Past, present and future challenges. Methods Inf Med 2004; 43(4): 308-14.

2. Linnenbrügger NI, Webber RL, Kobbelt LP, Lehmann TM. Automated hybrid TACT® volume reconstructions. Methods Inf Med 2004; 43(4): 315-9.

3. Weber S, Schüle T, Schnörr C, Hornegger J. A linear programming approach to limited angle 3D reconstruction from DSA projections. Methods Inf Med 2004; 43(4): 320-6. 
Lehmann et al.

4. Fischer G, Pfeifer B, Seger M, Hintermüller C, Hanser F, Modre R, Tilg et al. Computationally efficient noninvasive cardia activation imaging. Methods Inf Med 2005; 44(5): 674-86.

5. Cerutti S, Bianchi AM, Mainardi LT. Advanced spectral methods for detecting dynamic behavior. Autonomic Neuroscience-Basic \& Clinical 2001; 90: 3-12.

6. Winterhalder M, Schelter B, Hesse W, Schwab K, Leistritz L, Klan D, et al. Comparison of time series analysis techniques to detect direct and time-varying interrelations in multivariate, neural systems. Signal Processing Journal 2005; 85: 2137-60.

7. Ganesan R, Das TK, Venkataraman V, Waveletbased multiscale statistical process monitoring - A literature review. IIE Transactions on Quality and Reliability Engineering 2004; 36(9): 787-806.

8. Cohen L. Time-frequency analysis. IEEE Signal Processing Magazine 1999; 16: 22-8.

9. Lehnertz K, Andrzejak RG, Arnhold J, Kreuz T, Mormann F, Rieke Widman C, et al. Nonlinear EEG analysis in epilepsy: its possible use for interictal focus localization, seizure anticipation, and prevention. J Clin Neurophysiol 2001; 18: 209-22.

10. Rapp PE, Watanabe TAA, Faure P, Cellucci CJ. Nonlinear signal classification. International Journal of Bifurcation and Chaos 2002; 12: 1273-93.

11. Mulert C, Jager L, Schmitt R, Bussfeld P, Pogarell $\mathrm{O}$, Moller HJ, et al. Integration of fMRI and simultaneous EEG: towards a comprehensive understanding of localization and time-course of brain activity in target detection. Neuroimage 2004; 22: 83-94.

12. Xiao X, Mullen TJ, Mukkamala R. System identification: a multi-signal approach for probing neural cardiovascular regulation. Physiological Measurement 2005; 26: 41-71.

13. Coiera EW. Automated signal interpretation. In: Hutton RP, editor. Monitoring in Anesthesia and Intensive Care. London: W. B. Saunders Co. Ltd. 1994. p. 32-42.

14. Celka P, Boashash B, Colditz P. Preprocessing and time-frequency analysis of newborn EEG seizures. IEEE Engineering in Medicine and Biology Magazine 2001; 20: 30-9.

15. Agarwal R, Gotman J. Long-term EEG compression for intensive-care settings. IEEE Eng Med Biol Mag 2001; 20: 23-9.

16. Thoraval L, Carrault G, Schleich JM, Summers R, van de Velde M, Diaz J. Data fusion of electrophysiological and haemodynamic signals for ventricular rhythm tracking IEEE Eng Med Biol Mag 1997; 16: 48-55.

17. Stokking R, Zubal IG, Viergever MA. Display of fused images: Methods, interpretation, and diagnostic improvements. Semin Nucl Med 2003; 33: 219-27.

18. Lohscheller J, Dollinger M, Schuster M, Schwarz R, Eysholdt U, Hoppe U. Quantitative investigation of the vibration pattern of the substitute voice generator. IEEE Trans Biomed Eng 2004; 51: 1394-400.

19. Sintchenko V, Coiera EW. Which clinical decisions benefit from automation? A task complexity approach. Int J Med Inform 2003; 70: 309-16.

20. Coiera EW. Artificial intelligence in medicine: the challenges ahead. J Am Med Inform Assoc 1996; 3 : 363-6.

21. Petersson KM, Nichols TE, Poline JB, Holmes AP. Statistical limitations in functional neuroimaging I. Non-inferential methods and statistical models. Philosophical Transactions of the Royal Society of London Series B-Biological Sciences 1999; 354: 1239-60.

22. Reichertz PL. Towards systematisation. Methods Inf Med 1977; 16(3): 125-30.

23. Haux R. On medical informatics. Methods Inf Med1989; 28: 66-8.

24. Kalender W. Computed Tomography. Fundamentals, System technology, Image Quality, Applications. New York: Wiley \& Sons; 2001.

25. Hsieh J. Computed Tomography. Principles, Design, Artifacts, and Recent Advances. SPIE Press, Bellingham; 2003.

26. Becker CR, Ohnesorge BM, Schoepf UJ, Reiser MF. Current development of cardiac imaging with multidetector-row CT. Eur J Radiol 2000; 36: 97-103.

27. Imhof H, Schibany N, Ba-Ssalamah A, Czerny C, Hojreh A, Kainberger F, et al. Spiral CT and radiation dose. Eur J Radiol 2003; 47: 29-37.

28. Aach T, Schiebel U, Spekowius G. Digital image acquisition and processing in medical $\mathrm{x}$-ray imaging. J Electron Imaging 1999; 8: 7-22.

29. Giacomuzzi SM, Erckert B, Freund MC, Schopf T, Dessl A, Jaschke W. Dose reduction in computerized tomography with a new scan procedure. Aktuelle Radiologie 1996; 6: 110-3.

30. Kopka L, Funke M, Breiter N, Hermann KP, Vosshenrich R, Grabbe E. An anatomically adapted variation of the tube current in $\mathrm{CT}$ - Studies on radiation dosage reduction and image quality. Fortschritte auf dem Gebiete der Röntgenstrahlen und der Nuklearmedizin 1995; 163(5): 383-7.

31. Hundt W, Rust F, Stäbler A, Wolff H, Suess C, Reiser M. Dose reduction in multisclice computed tomography. J Comput Assist Tomogr 2005; 29(1): 140-7.

32. Feldkamp LA, Davies LC, Kress JW. Practical cone-beam algorithm. J Opt Soc Am A 1984; 1: $612-9$.

33. Xiao S, Bresler Y, Munson Jr. DC. Fast feldkamp algorithm for cone-beam computer tomography. Proceedings IEEE International Conference on Image Processing (ICIP) 2003; 819-22.

34. Spekowius G, Boerner H, Eckenbach W, Quadflieg P, Laurenssen GJ. Simulation of the imaging performance of $x$-ray image intensifier/ TV camera chains. Proceedings SPIE 1995; 2432: 12-23.

35. Rabbani M, Shaw R, van Mettler R. Detective quantum efficiency of imaging systems with amplifying and scattering mechanisms. Journal Opt Soc Am A 1987; 4(5) :895-901.

36. Koppe R, Klotz E, op de Beek JO, Aerts H. 3D vessel reconstruction based on rotational angiography. Proceedings Computer Assisted Radiology (CAR) 1995; 101-7.

37. Busse F, Rütten W, Sandkamp B, Alving PL, Bastiaens R. Design and performance of a highquality cardiac flat panel detector. Proceedings SPIE 2002; 4682: 819-27.

38. Siewerdsen JH, Jaffray DA. Optimization of X-ray imaging geometry (with specific application to flatpanel cone-beam computed tomography). Med Phys
2000; 27: 1903-14.

39. Siewerdsen JH, Jaffray DA. Cone-beam computed tomography with a flat-panel imager: Magnitude and effects of x-ray scatter. Med Phys 2001; 28: $220-31$.

40. Rose G, Wiegert J, Schaefer D, Fiedler K, Conrads $\mathrm{N}$, Timmer J, et al. Image quality of flat panel conebeam CT. Proceedings SPIE 2003; 5030: 677-83.

41. Bertram M, Rose G, Schäfer D, Wiegert J, Aach T. Directional interpolation of sparsely sampled conebeam CT sinogram data. Proceedings IEEE International Symposium on Biomedical Imaging (ISBI) 2004; 928-31.

42. Bigün J, Granlund GH. Optimal orientation detection of linear symmetry. Proceedings IEEE International Conference on Computer Vision (ICCV) 1987; 433-8.

43. Di Zenzo S. A note on the gradient of a multiimage. Computer Vision, Graphics, and Image Processing 1986; 33: 116-25.

44. Lehmann TM, Gönner C, Spitzer K: Survey: Interpolation methods in medical image processing. IEEE Trans Med Imaging 1999; 18(11): 1049-75.

45. Lehmann TM, Gönner C, Spitzer K: Addendum B-spline interpolation in medical image processing. IEEE Trans Med Imaging 2001; 20(7): 660-5.

46. Endo M, Tsunoo T, Nakamori N. Effect of scatter radiation on image noise in cone beam $\mathrm{CT}$. Proceedings SPIE 2000; 3977: 514-21.

47. Wiegert J, Aach T, Rose G, Schaefer D, Bertram M, Conrads N, et al. Performance of standard fluoroscopy anti-scatter grids in flat panel based cone beam CT. Proceedings SPIE 2004; 5368: 67-78.

48. Siewerdsen JH, Mosley DJ, Bakhtiar B, Richard S, Jaffray DA. The influence of antiscatter grids on soft-tissue detectability in cone-beam computed tomography with flat-panel detectors. Med Phys 2004; 31: 3506-20.

49. Wiegert J, Bertram M, Rose G, Aach T. Model based scatter correction for cone-beam computed tomography. Proceedings SPIE 2005; 5745: 271-82.

50. Seidel G, Meyer K, Berdien G, Hollstein D, Toth $\mathrm{D}$, Aach T. Ultrasound perfusion imaging in acute middle cerebral artery infarction predicts stroke outcome. Stroke 2004; 35: 1107-11.

51. Wei K, Jayaweera A, Firoonza S, Linka A, Skyba D, Kaul S. Quantification of myocardial blood flow with ultrasound-induced destruction of microbubbles administered as a constant venous infusion. Circulation 1998; 97: 473-83.

52. Schoelgens C. Native tissue harmonic imaging. Radiologe 1998; 5: 420-423.

53. Kier C, Toth D, Meyer-Wiethe K, Schindler A, Seidel G, Aach T. Cerebral perfusion imaging with bolus harmonic imaging. Proceedings SPIE 2005; 5750: 437-46.

54. Krakow K, Allen PJ, Lemieux L, Symms MR, Fish DR. Methodology: EEG-correlated fMRI. Adv Neurol 2000; 83: 187-201.

55. Tzabazis A, Ihmsen H, Schywalsky M, Schwilden H. EEG-controlled closed-loop dosing of propofol in rats. Br J Anaesth 2004; 92: 564-9.

56. Sinkjaer T, Haugland M, Inmann A, Hansen M, Nielsen KD. Biopotentials as command and feedback signals in functional electrical stimulation systems. Medical Eng Phys 2003; 25: 29-40.

57. Roco MC, Bainbridge WS. Converging 
technologies for improving human performance. Dordrecht: Kluwer Academic Publishers; 2003.

58. Bach-y-Rita P, Kercel SW. Sensory substitution and the human-machine interface. Trends in Cognitive Sciences 2003; 7: 541-6.

59. del R. Millan J, Renkens F, Mourino J, Gerstner W. Noninvasive brain-actuated control of a mobile robot by human EEG. IEEE Trans Biomed Eng 2004; 51: $1026-31$

60. Santana D, Ramirez M, Ostrosky-Solis F. Recent advances in rehabilitation technology: A re-view of the brain-computer interface. Revista De Neurologia 2004; 39: 447-50.

61. Bruns A. Fourier-, Hilbert- and wavelet-based signal analysis: are they really different approaches? J Neurosci Methods 2004; 137: 321-32.

62. Nie K, Stickney G, Zeng FG. Encoding frequency modulation to improve cochlear implant performance in noise. IEEE Trans Biomed Eng 2005; 52: 64-73.

63. Flanagan LJ, Golden RM. Phase vocoder. The Bell System Technical Journal 1966; 45: 1494-509.

64. Niblack W, Barber R, Equitz W, Flickner M, Yanker P, Ashley J. The QBIC-project - Querying images by content using color, texture and shape. Proceedings SPIE 1993; 1908: 173-87.

65. Flickner M, Sawhney H, Niblack W, Ashley J, Huang Q, Dom B, et al. Query by image and video content - The QBIC system. IEEE Computer 1995; 28(9): 23-32.

66. Tagare HD, Jaffe CC, Duncan J. Medical image databases - A content-based retrieval approach. J Am Med Inform Assoc 1997; 4: 184-98.

67. Müller H, Michoux N, Bandon D, Geissbuhler A. A review of content-based image retrieval systems in medicine-Clinical benefits and future directions. Int J Med Inform 2004; 73: 1-23.

68. Smeulders AWM, Worring M, Santini S, Gupta A, Jain R. Content-based image retrieval at the end of the early years. IEEE Transactions on Pattern Analysis and Machine Intelligence 2000; 22(12): 1349-80.

69. Lehmann TM, Güld MO, Thies C, Fischer B, Spitzer K, Keysers D, Ney et al. Content-based image retrieval in medical applications. Methods Inf Med 2004; 43(4): 354-61

70. Lehmann TM, Schubert H, Keysers D, Kohnen M, Wein BB. The IRMA code for unique classification of medical images. Proceedings SPIE 2003; 5033:
440-51.

71. Lehmann TM, Güld MO, Deselaers T, Keysers D, Schubert H, Spitzer K, et al. Automatic categorization of medical images for content-based retrieval and data mining. Computerized Medical Imaging and Graphics 2005; 29(2): 143-52.

72. Haralick RM, Shanmugam K, Dinstein I. Textural features for image classification. IEEE Trans Syst Man Cybern 1973; 3: 610-21.

73. Tamura H, Mori S, Yamawaki T: Textural features corresponding to visual perception. IEEE Trans Syst Man Cybern 1978; 8(6): 460-72.

74. Güld MO, Keysers D, Leisten M, Schubert H, Lehmann TM. Comparison of global features for categorization of medical images. Proceedings SPIE 2004; 5371: 211-22

75. Keysers D, Dahmen J, Ney H, Wein B, Lehmann TM. A statistical framework for model-based image retrieval in medical applications. J Electron Imaging 2003; 12(1): 59-68.

76. Lehmann TM, Güld MO, Keysers D, Schubert H, Kohnen M, Wein BB. Determining the view position of chest radiographs. J Digit Imaging 2003; 16(3): 280-91.

77. Pan B, Zhang D, Li N, Wang K. Computerized tongue diagnosis based on Bayesian networks. IEEE Trans Biomed Eng 2004; 51(10): 1803-10.

78. Chiu CC, Lin HS, Lin SL. A structural texture recognition approach for medical diagnosis through tongue. Biomedical Engineering, Applications, Basis and Communications 1995; 7(2): 143-8.

79. Chiu CC. A novel approach based on computerized image analysis for traditional Chinese medical diagnosis of the tongue. Comput Methods Programs Biomed 2000; 61(2): 77-89.

80. Li CH, Yuen PC. Tongue image matching using color content. Pattern Recognition 2002; 35(2): 407-19.

81. Fischer B, Modersitzki J. Intensity based image registration with a guaranteed one-to-one point match. Methods Inf Med 2004; 43(4): 327-30.

82. Bartz D, Orman J, Gürvic Ö. Accurate volumetric measurements of anatomic cavities. Methods Inf in Med 2004; 43(4): 331-5.

83. Chrástek R, Skokan M, Kubecka L, Wolf M, Donath $\mathrm{K}$, Jan J, et al. Multimodal retinal image registration for optic disk segmentation. Methods Inf Med 2004; 43(4): 336-42.

84. Wittenberg T, Grobe M, Münzenmayer C, Kuziela
H, Spinner K. A semantic approach to segmentation of overlapping objects. Methods Inf Med 2004; 43(4): 343-53.

85. Imabayashi E, Matsuda H, Asada T, Ohnishi T, Sakamoto S, Nakano S, et al. Superiority of 3dimensional stereotactic surface projection analysis over visual inspection in discrimination of patients with very early Alzheimer's disease from controls using brain perfusion SPECT. J Nucl Med 2004; 45(9): 1450-7.

86. Burdette JH, Minoshima S, Borght TV, Tran DD, Kuhl DE. Alzheimer Disease - Improved visual inspection of PET images by three-dimensional stereotaxic surface projections. Radiology 1996; 198: 837-43.

87. Minoshima S, Giordani B, Berent S. et al. Metabolic reduction in the posterior cingolate cortex in very early Alzheimer's disease. Ann Neurol 1997; 42: 85-94.

88. Gerig G, Koller T, Szekely G, Brechbühler C, Kübler O. Symbolic description of 3-D structures applied to cerebral vessel tree obtained from MR angiography volume data. Lecture Notes in Computer Science 1993; 94-111.

89. Selle D, Preim B, Schenk A, Peitgen HO. Analysis of vaslulature for liver surgical planning. IEEE Transactions of Medical Imaging 2002; 21(11): 1344-57.

90. Bloomenthal J, Shoemake K. Convolution surfaces. Computer Graphics 1991; 25(4): 251-6.

91. Oeltze S, Preim B. Visualization of vasculature with convolution surfaces - Method, validation and evaluation. IEEE Trans Med Imaging 2005; 24(4): 540-8.

92. Suzuki S, Suzuki N, Hattori A, Uchiyama A, Kobayashi S. Sphere-filled organ model for virtual surgery system. IEEE Trans Med Imaging 2004; 23(6): 714-22.

Correspondence to:

Thomas M. Lehmann

RWTH Aachen

Institut für Medizinische Informatik

D.52027 Aachen

Germany

E-Mail: lehmann@ computer.org 